J. theor. Biol. (1976) 61, 185-193

\title{
A Physical Model for the Simultaneous Membrane Transport and Metabolism of Drugs
}

\author{
N. F. H. Ho, J. Park, W. Morozowich and W. I. Higuchi \\ College of Pharmacy, The University of Michigan, \\ Ann Arbor, Michigan and \\ Pharmacy Research, The Upjohn Company, \\ Kalamazoo, Michigan, U.S.A.
}

(Received 17 April 1975, and in revised form 2 November 1975)

A simple model for the simultaneous passive membrane transport and bioconversion of a drug, which may be a weak electrolyte or a neutral molecule, is mathematically described. It includes an aqueous diffusion layer and an operational aqueous pore pathway. The applicability of the model is shown for the in situ rat intestinal transport of prostaglandin $F_{2 a}$.

\section{Introduction}

The occurrence of the simultaneous membrane transport and bioconversion of drugs in the intestinal tract comes as no surprise in view of the fact that many enzyme systems are found on the membrane surface and within the intestinal membrane and enterocyte. In quantitative and mechanistic studies on the simultaneous transport and bioconversion of solute molecules across the intestinal barrier, a path length of approximately $20 \mu \mathrm{m}$ from the mucosal surface to the blood capillary bed, it is prerequisite that one distinguishes between cavital, membrane and intracellular digestion. Ugolev $(1968,1974)$ and Crane (1975) have expressed various viewpoints on membrane digestion in dealing, particularly, on the digestion of carbohydrates. However, the broad implication with respect to solute molecules such as drugs is that there are two possible membrane metabolic situations for consideration. One is the existence of surface-bound enzymes oriented toward the aqueous environment of the intestinal lumen leading to membrane contact digestion. Here, the enzyme systems found in the glycocalyx are also included. The other is the location of the relevant enzyme system within the membrane.

Using salicylamide as the model substrate, Barr \& Riegelman (1970) studied the intestinal salicylamide glucuronide formation and transport in the rabbit with in vitro cannulated everted intestines and in vivo perfused 
closed loops with complete mesenteric venous blood collection and examined some theoretical physical models. Magee, Armour \& Miller (1973) showed that the absorption of the prostaglandin $\mathrm{F}_{2 \alpha}$ and $\mathrm{E}_{2}$ acids and the 15-methyl$F_{2 \alpha}$ methyl ester is accompanied by extensive metabolism by the small intestines of the rat. Similar results were found in absorption experiments using intact intestinal loops of dogs (Robert, Magee, Miller \& Nezamis, 1974). Using $\left[1-{ }^{14} \mathrm{C}\right]$ prostaglandin $\mathrm{E}_{1}$, Parkinson \& Schneider (1969) found that only minute amounts of the intact weak acid survived the transport from the mucosal to the serosal fluid of rat jejunum segments in vitro. They attributed the loss of the ${ }^{14} \mathrm{C}$-label to an oxidative process leading to the formation of carbon dioxide. Prostaglandin $\mathrm{E}_{1}$ is metabolized by homogenates of both rat and dog stomach and jejunum (Nakano, Prancan \& Morsy, 1973).

The purpose of this paper is to present a simple physical model for the simultaneous drug transport and bioconversion across the intestinal membrane. The model is derived fiom well-established physicochemical concepts with the accompanying mathematics and is described in such a manner that it provides the basis for the design of experiments and interpretation of data for obtaining quantitative estimates of meaningful phenomenological parameters. Specifically, the model is directed to the intestinal transport of the natural prostaglandins.

\section{General Description of the Physical Model}

The physical model for the simultaneous transport and bioconversion of a drug, taken here as a weak acid, is shown in Fig. 1. It consists of an aqueous diffusion layer (often referred to as the unstirred aqueous layer) in the lumen in series with the intestinal membrane barrier followed by the blood sink. Operationally, the membrane is comprised of two parallel pathways: the simultaneous diffusional transport-bioconversion pathway in the lipoidal fraction of the membrane and the aqueous pore pathway. It is assumed that (a) quasi-steady state conditions prevail, (b) metabolism in the lumenal solution, if it occurs, is insignificant, (c) only non-dissociated drug species are able to partition into the lipoidal fraction of the membrane, (d) metabolism occurs homogeneously within the membrane and, consequently, no significant metabolism is assumed to occur with membrane surface enzymes, (e) the transport of non-dissociated and anionic drug species across the aqueous pores occurs with equal facility, (f) iso-osmotic conditions exist everywhere, (g) the aqueous diffusion coefficients of non-dissociated and anionic drug species are identical and (h) sink conditions prevail on the blood side. It is 


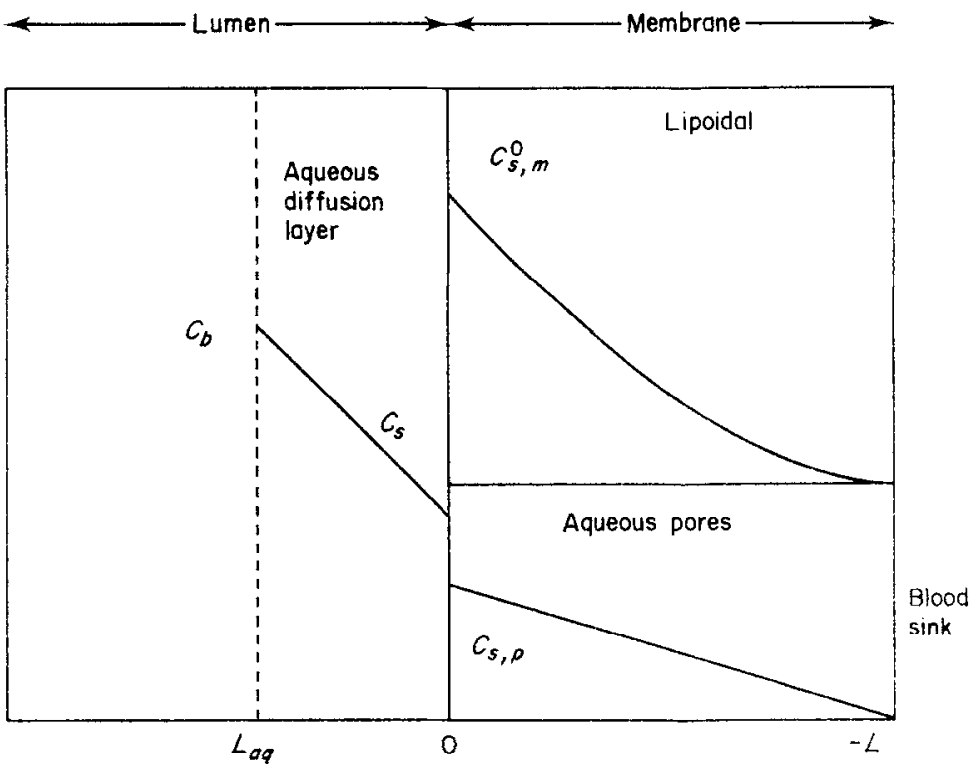

FIG. 1. Physical model for the simultaneous intestinal membrane transport and bioconversion of a substrate drug. An aqueous diffusion layer in the intestinal lumen is in series with the membrane consisting of two parallel pathways: the diffusional transport-bioconversion pathway and the aqueous pore pathway. The model depicts the boundary concentrations and distances.

also taken here that since the radii of curvature of the aqueous diffusion layer and the membrane are not too different from each other, the planar model is applicable (Flynn, Ho \& Higuchi, 1976).

\section{Steady-State Fluxes}

The total flux of the drug (weak acid) across the aqueous diffusion layer is

$$
J_{a q}=\frac{D_{a q}}{L_{a q}}\left(C_{b}-C_{s}\right)=P_{a q}\left(C_{b}-C_{s}\right),
$$

$J_{a g}=$ total flux in the aqueous diffusion layer per unit area.

$D_{a q}=$ aqueous diffusion coefficient, $\mathrm{cm}^{2} \mathrm{~s}^{-1}$.

$L_{a q}=$ effective thickness of the aqueous diffusion layer.

$P_{a q}=$ permeability coefficient $\left(=D_{a q} / L_{a q}\right), \mathrm{cm} \mathrm{s}^{-1}$.

$C_{b}, C_{s}=$ total concentration of non-dissociated and anionic species in the bulk lumenal solution and at the membrane surface. 
The quasi-steady state change in the concentration in the membrane with distance $x$ is given by the following expression which describes the simultaneous diffusional transport and homogenous metabolism:

$$
D \frac{\mathrm{d}^{2} C}{\mathrm{~d} x^{2}}-k C=0 \quad(0 \geq x \leq-L)
$$

$D=$ diffusion coefficient in the membrane, $\mathrm{cm}^{2} \mathrm{~s}^{-1}$.

$k=$ apparent first-order enzyme rate constant, $\mathrm{s}^{-1}$.

$C=$ concentration in the membrane.

$L=$ thickness of the membrane.

With the boundary conditions,

$$
\begin{aligned}
& C=C_{s, m}^{0} \text { at } x=0 \\
& C=0 \text { at } x=-L
\end{aligned}
$$

the solution to equation (2) is

$$
C=\frac{C_{s, m}^{0} \sinh [\sqrt{k / D}(L+x)]}{\sinh (L \sqrt{k / D})}
$$

where $C_{s, m}^{0}=$ concentration of non-dissociated species at the surface on the membrane side. Thus, the concentration of the intact drug in the membrane decreases exponentially with distance. The pathway of the membrane flux across the diffusional transport-bioconversion is obtained by integrating equation (2) once; accordingly,

$$
\text { constant }=D \frac{\mathrm{d} C}{\mathrm{~d} x}+k \int C \mathrm{~d} x
$$

Using equation (3),

$$
J_{m}=\frac{\alpha C_{s, m}^{0} \sqrt{k D} \cosh [\sqrt{k / D}(L+x)]}{\sinh (L \sqrt{k / D})}
$$

$J_{m}=$ flux in the membrane per unit apparent surface area.

$C_{s, m}^{0}=$ concentration of non-dissociated species at the surface on the membrane side.

$\alpha=$ volume fraction of lipoidal phase of the membrane.

The flux of the total concentration of drug per unit area across the aqueous pores of the membrane into the blood sink is

$$
J_{p}=\frac{(1-\alpha) D_{p} C_{s}}{L}=P_{p} C_{s},
$$

$J_{p}=$ flux per unit apparent surface area across the pores. 
$(1-\alpha)=$ volume fraction of aqueous pores of the membrane.

$D_{p}=$ diffusion coefficient in the pores, $\mathrm{cm}^{2} \mathrm{~s}^{-1}$.

$L=$ thickness of the membrane.

$P_{p}=$ permeability coefficient of the pores $\left[=(1-\alpha) D_{p} / L\right] \mathrm{cm} \mathrm{s}^{-1}$.

Here, it is taken that there is no metabolism occurring in the aqueous pore pathway.

The continuity of flow across the membrane interface is

$$
J_{a q}=\left(J_{m}\right)_{x=0}+J_{p} .
$$

The lipoidal membrane-water partition coefficient is

$$
K=\frac{C_{s, m}^{0}}{C_{s}^{0}}=\frac{C_{s, m}^{0}}{C_{p}^{0}}
$$

$K=$ partition coefficient.

$C_{\mathrm{S}}^{0}=$ concentration of non-dissociated species at the membrane surface on the lumen side.

The fraction of non-dissociated species $\uparrow$ of the weak acid is given by

$$
X_{s}=\frac{C_{s}^{0}}{C_{s}}=\frac{1}{1+K_{\mathrm{a}} /\left(H^{+}\right)_{s}}
$$

$X_{s}=$ fraction of non-dissociated species at the membrane surface.

$K_{a}=$ dissociation constant.

$\left(\mathrm{H}^{+}\right)_{s}=$ hydrogen ion concentration at membrane surface.

After combining equations (1) and (5)-(9) and algebraic rearrangement, the total concentration at the membrane surface on the lumen side is explicitly related to the total concentration of drug in the bulk lumenal solution by

$$
C_{s}=\frac{P_{a q} C_{b}}{P_{a q}+\frac{\alpha K X_{s} \sqrt{k D}}{\tanh (L \sqrt{k / D})}+P_{p}}
$$

whereupon equation (1) becomes

$$
J_{a q}=\frac{C_{b}}{\frac{1}{P_{a q}}+\frac{1}{\frac{\alpha K X_{s} \sqrt{k D}}{\tanh (L \sqrt{k / D})}+P_{p}}}
$$

Since

$$
J_{a q}=-\frac{V}{A} \frac{\mathrm{d} C_{b}}{\mathrm{~d} t}
$$

$\uparrow$ For a weak base, $X_{s}-1 /\left(1+\left(H^{+}\right)_{s} / K_{a}\right)$ and for a neutral molecule, $X_{s}=1$. 
$V=$ volume of solution.

$A=$ apparent surface area.

$t=$ time.

then

$$
\frac{\mathrm{d} C_{b}}{\mathrm{~d} t}=-K_{u} C_{b}
$$

where

$$
K_{u}=\frac{A}{V} \cdot \frac{1}{\frac{1}{P_{a q}}+\frac{1}{\frac{\alpha K X_{s} \sqrt{k D}}{\tanh (L \sqrt{k / D})}+P_{p}}}
$$

Integrating between the initial total concentration $C_{b}(0)$ and $C(t)$ one obtains the semi-logarithmic expression:

$$
\ln C_{b}=\ln C_{b}(0)-K_{u} t .
$$

\section{Special Case of Simple Passive Transport}

In the special case when there is an insignificant occurrence or absence of bioconversion during the passive transport across the membrane, i.e. $k \ll D$, the $\tanh (L \sqrt{k / D})$ term in equation (14) converges to zero, and consequently, gives an apparent impression that there is no passive membrane transport. Physically, this is inconsistent. However, letting $u=L \sqrt{k / D} \ll 1 \cdot 0$, the series expansion leads to

$$
\tanh u \simeq u+\ldots \quad(u \ll 1 \cdot 0)
$$

in which the higher terms are small and inconsequential. It follows that

$$
\frac{\lim _{u \rightarrow 0} \tanh u}{\sqrt{k D}} \simeq \frac{L}{D}
$$

and also equation (14) reduces to a familiar expression (Ho, Higuchi \& Turi, 1972):

$$
K_{u}=\frac{A}{V} \cdot \frac{1}{\frac{1}{P_{a q}}+\frac{1}{\frac{\alpha K}{L} X_{s} D}+P_{p}}
$$




\section{Factorization of the Effective Transport-bioconversion Permeability Coefficient}

It is possible to gain a further quantitative insight into the simultaneous diffusion and enzymatic bioconversion phenomena in the membrane. Let the effective membrane passive transport-bioconversion permeability coefficient $\left(P_{e, m}^{\prime}\right)$ be defined by

$$
P_{e, m}^{\prime}=\frac{\alpha K X_{s} \sqrt{k D}}{\tanh (L \sqrt{k / D})}+P_{p}
$$

and the effective membrane passive transport permeability coefficient $\left(P_{e, m}\right)$ by

$$
P_{c, m}=\frac{\alpha K X_{s} D}{L}+P_{p}
$$

at a constant $\mathrm{pH}$, the ratio of equations (19) and (20) is

$$
\frac{\left(P_{e, m}^{\prime}-P_{p}\right)}{\left(P_{e, m}-P_{p}\right)}=\frac{L \sqrt{k / D}}{\tanh (L \sqrt{k / D})}
$$

from which the term $L \sqrt{k / D}$ is readily calculated since $P_{e, m}^{\prime}, P_{e, m}$ and $P_{p}$ are experimentally determined coefficients. The substitution of this calculated term into the argument of the tanh function in equation (19) leads to

$$
\alpha K \sqrt{k D}=\frac{\left(P_{e, m}^{\prime}-P_{p}\right) \tanh (L \sqrt{k / D})}{X_{s}}
$$

where $\alpha K \sqrt{k D}$, having units of $\mathrm{cm} \mathrm{s}^{-1}$, is physically the intrinsic membrane transport-bioconversion permeability coefficient. The experimental approach in accomplishing the factorization involves the carrying out of absorption studies in the absence and presence of relevant enzyme inhibitors.

\section{Relevance of the Physical Model to Absorption Experiments}

The physical model predicts that the total concentration of the weak acid drug in the intestinal lumen decreases logarithmically with time. As seen in equation (14), the slope will be influenced by the buffer $\mathrm{pH}$ of the solution and the hydrodynamics of the solution in the lumen. At low pH $\left(<\mathrm{p} K_{a}\right)$ the concentration of non-dissociated species is high and the rate constant will be larger as compared to that at a higher $\mathrm{pH}$. However, when the $\mathrm{pH} \gg \mathrm{p} K_{a}$, the rate constant will attain a minimum asymptotic value operationally indicative of the transport of the anionic species across the aqueous pores of the membrane. Because of the influence of the hydrodynamics on the effective thickness of the aqueous diffusion layer $\left(L_{a q}\right)$, 
the permeability coefficient of the aqueous boundary layer $\left(P_{a q}\right)$ will be larger when the solution is agitated than the corresponding permeability coefficient when the solution is less agitated. Consequently, the absorption rate will be faster with higher degrees offagitation under the conditions when the total absorption rate is at least $25 \%$ influenced by diffusion across the unstirred aqueous layer in front of the membrane. It is expected that the degree of hydrodynamics will have an insignificant effect on the absorption rate of an essentially membrane-controlled situation.

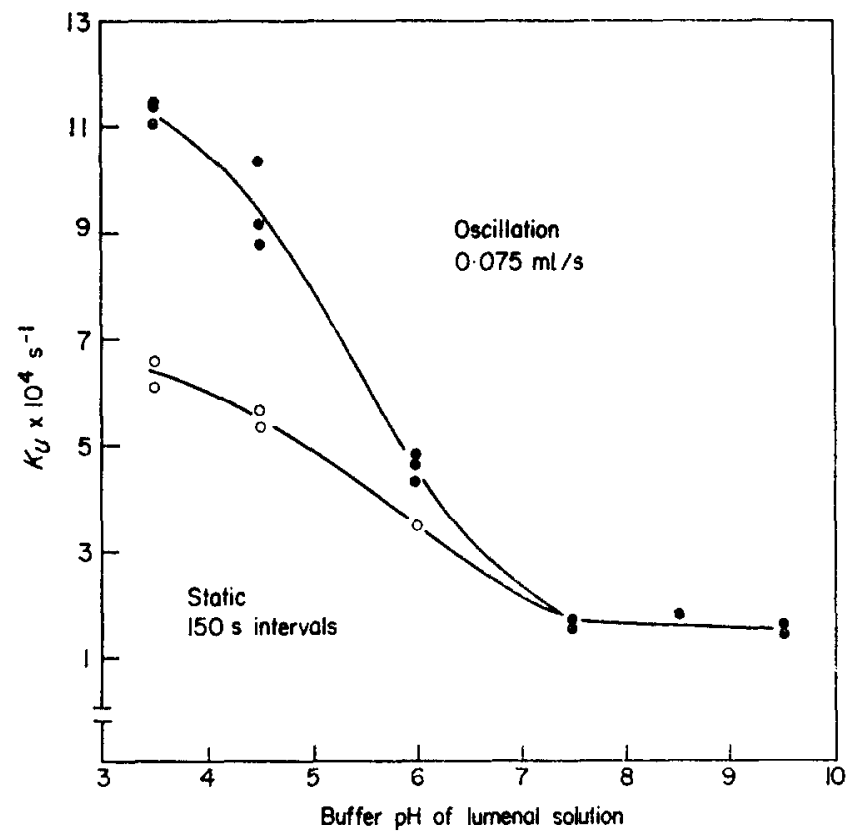

FIg. 2. Apparent first order absorption rate constant versus buffer $\mathrm{pH}$ profiles of prostaglandin $F_{2 \alpha}$ at two different hydrodynamic conditions from in situ absorption experiments in the rat jejunum.

The experimental evidence for the physical model described herein is shown in Fig. 2. It involves the simultaneous transport and metabolism of prostaglandin $\mathrm{F}_{2 \alpha}\left(\mathrm{PGF}_{2 \alpha}\right)$ across the jejunal membrane of the rat using an in situ non-perfusion techniquet. The apparent first-order absorption rate constant $K_{u}$ versus $\mathrm{pH}$ profiles at two hydrodynamic situations show the physical interactions between the $\mathrm{pH}$ and permeabilities of the aqueousdiffusion

$\uparrow$ Manuscript on the experimental description and data analysis for factorization of the physical transport parameters is in progress. 
layer, passive transport-bioconversion pathway and aqueous pores. Qualitative analyses of the plasma fraction of blood from the portal vein by high pressure liquid chromatography and thin layer chromatography indicate the presence of 15-keto-PGF $2 \alpha$ and 15-keto-13,14-dihydro-PGF ${ }_{2 \alpha}$ and the absence of $\mathrm{PGF}_{2 x}$. Experimental studies are continuing with other natural prostaglandins and their esters.

\section{Summary}

A physical model for the intestinal transport of solutes has been described in which an aqueous diffusion layer barrier is in series with the membrane barrier consisting of a passive diffusional-bioconversion pathway and an operational aqueous pore pathway in parallel. It provides the basis for the experimental design of in situ studies and quantification of physically meaningful and experimentally accessible transport and overall bioconversion kinetic parameters. The interaction and factorization of (a) the permeability coefficients of the aqueous diffusion layer, aqueous pores of the membrane and the principal membrane transport-bioconversion pathway and (b) the $\mathrm{pH}$ and $\mathrm{p} K_{a}$ and (c) the lipophilicity of the solute are mathematically explicit. The applicability of the model is shown with preliminary in situ rat intestinal absorption studies with prostaglandin $F_{2 \alpha}$.

\section{REFERENCES}

Barr, W. H. \& Riegelman, S. (1970). J. Pharm. Sci. 59, 164.

CRANE, R. K. (1975). In Intestinal Absorption and Malabsorption (T. Z. Csaky, ed.) p. 127. New York: Raven Press.

FlynN, G. L., Ho, N. F. H. \& Higuchi, W. I. (1976). J. Pharm. Sci., 65, 154.

Ho, N. F. H., Higuchi, W. I. \& TuRI, J. (1972). J. Pharm. Sci. 61, 192.

Magee, W. E., Armour, S. B. \& Miller, O. V. (1973). Biochim. Biophys. Acta 306, 270.

Nakano, J., Prancan, A. V. \& Morsy, N. H. (1973). Japan J. Pharmacol. 23, 355.

Parkinson, T. M. \& Schneider, J. C. (1969). Biochim. Biophys. Acta 176, 78.

Robert, A., Magfe, W. E., Mil..fr, O. V. \& Nfzamis, J. F. (1974). Biochim. Biophys. Acta 348, 269.

Ugolev, A. M. (1968). Physiology and Pathology of Membrane Digestion. New York: Plenum Press.

Ugolev, A. M. (1974). In Biomembranes: Intestinal Absorption, vol, 4A, Ch. 7 (D. H. Smyth, ed.). New York: Plenum Press. 